\title{
Object Motion Direction Detection and Tracking for Automatic Video Surveillance
}

\author{
Adithya Urs ${ }^{1}$, Dr. Nagaraju $C^{2}$ \\ 1 Student Dept. Of ECE, 2 Assistant professor, Dept of ECE, The National Institute of Engineering, Mysuru, India \\ Email: adithya.s.urs@gmail.com
}

Received: 20 July 2020; Accepted: 06 September 2020; Published: 08 April 2021

\begin{abstract}
In today's world having a smart reliable surveillance system is very much in need. In fact in many public places like banks, jewellery stores, malls, schools and colleges it is basic necessary to have a surveillance system (CCTV). Most of today's implementations are not smart and they record videos during night even when there is no motion. This will lead to unnecessary storage usage and difficult to get the important part of the footage. And also, most of the today's implementations are stationary, they can't track the moving object. This report will outline a naive approach to implement a smart video surveillance system using object motion detection and tracking. Here we are using conventional Background subtraction model to detect motion and we estimate the direction of motion of object by comparing the centroid of the moving object in subsequent frames and track the moving object by rotating the camera using servo. Video recording takes place only when there is movement in the frame which helps in storage efficiency. We are also improving the speed of email alert delivery by using multithreading.
\end{abstract}

Index Terms: Background subtraction Contours, Convolution, Dilation, Erosion, Gaussian blur, Multithreading.

\section{Introduction}

Security has become a major concern in third millennium. Due to the increasing population and business of cities it is very difficult to monitor each and every person. At night monitoring the activities of each and every room in a huge building manually is difficult and tedious task. Here we are presenting a novel approach of motion tracking and object detection in video footage which take action based on the motion of any foreign objects into the video frame and also tracks the direction of movement of the object and move accordingly. In most part of the world they relay manually for intrusion detection. Some of the existing automatic surveillance systems use infrared sensors which detects intrusion by using two IR sensors in adjacent position and based on which sensor activates first the direction of motion can be predicted. This system can only detect motion but is highly unreliable when determining the direction of object

The other approach used today is by using PIR sensor. When the sensor is idle, both slots detect the same amount of IR, the ambient amount radiated from the room or walls or outdoors. When a warm body like a human passes by, it first intercepts one half of the PIR sensor, which causes a positive differential change between the two halves. When the warm body leaves the sensing area, the reverse happens, whereby the sensor generates a negative differential change. These change pulses are what is detected. The disadvantages of PIR sensors are they are easily interfered by heat sources. And sensitivity decreases when ambient temperature is equal to human body temperature

None of the existing automatic surveillance system can detect the identity of the intruder and also has the above Drawbacks.

Using CCTV can get the identification of the intruder but it needs continuous manual supervision. And also, the image based automatic intrusion detection system used today can detect motion but fails to automatically track the moving object.

Our approach is to use the camera footage and process it to detect direction of motion. And move the camera according to the direction of motion of the object. This is done by getting the directional information and steering the servo (which hosts the camera on top of it) using a micro-controller. We are also recording the footage only when object is detected which will increase storage efficiency. Speed of alerting is also improved by using multithreading over SMTP API.

Open CV is an open source library for image processing and Computer vision applications, is having the predefined library of various required essential operations as methods. So, we choose Open CV and Jupyter Notebook as the IDE for development. 


\section{Literature Survey} CV"

[1] Yongxiang $\mathrm{Wu}$, "Research on bank intelligent video image processing and monitoring system based on Open

This paper accomplishes the task of real time surveillance using human detection, Motion tracking and behavior judgement even here background subtraction method is used. The system developed can accurately judge motion and human detection helps in avoiding false alarms.

[2] Neha Gaba, Neelam Barak and shipra Aggarwal. "Motion Detection, Tracking and Classification for Video Surveillance"

Here frame differencing method is used in which background is modeled first and is used as a reference based on which motion is detected.

[3] Khirod Chandra Sahoo; Umesh Chandra Pati "IoT based intrusion detection system using PIR sensor".

Simple cost effective, Iot based always connected to internet so it is very effective in alerting. Use of PIR sensor helps in detecting even in dark environment. But fails to identify the intruder.

Each of the existing surveillance system detects motion but fails to identify the direction of motion and track the moving object. Our main research objective is to detect the direction of motion, also a storage efficient system. Our secondary objective is to improve the speed of email alert delivery in an intrusion detection system.

\section{Proposed Methodology}

Our approach for accomplishing the above objective is as follows, we are using background subtraction, which is quite simple model where two subsequent frames of video feed is subtracted to check for difference. This difference helps is detecting motion. On the differential frame we are applying Gaussian blur, Erosion and dilation to enhance the clarity of the moving object. The reasoning behind the usage of the above operations is given in the later section. After estimating the moving object, a contour is drawn around it. The centroid of the object is calculated. Now the relative position of the centroid of the object in subsequent frames gives the directional information. We are using a look-up table stored in the memory to map the angle of rotation of servo with the position of the object. Pyserial library is used to send the positional information of the object from the host computer to micro-controller through serial communication. Email alert delivery which is done by the host computer is improved by using multithreading to get real time performance.

\section{Hardware and Software}

1. Hardware Requirements:

Hardware we used to develop the module is as listed below.

Processor : Intel 8th gen i5

RAM : $\quad 8 \mathrm{~GB}+$

Hard disk space : : $1 \mathrm{~TB}+$.

Controller : $\quad$ Arduino UNO

Sensor : $\quad$ Standard 720p HD Camera

Steering device : Standard metal gear servo

2. Software Requirements:

Software required in development is as listed below

Operating System : : Windows 7 or Higher

Language : $\quad$ Python 3.7

Library : OpenCV

IDE

Com Protocol $\quad$ : Pyserial

\section{Algorithms}

The above model works on the principle background subtraction which is a very simple but also a very effective model at the same time. Background subtraction was used because it is computationally inexpensive can be run on portable devices and also gives fairly reasonable result.

\section{A. Background subtraction}

This is a quite simple model where subsequent frames of a video are captured and then they are converted to Grey scale and then corresponding pixel of first image is subtracted from the next image. After that each pixel of that corresponding image is compared to a per-set threshold value and if the value is greater than the threshold value it is 
considered as one else it is considered as zero by this way a binary image is formed. This binary image gives the data about the motion of object in the frame. The equation for the above operation is shown in equation 1 below.

$$
\mid \mathrm{P}[\mathrm{F}(\mathrm{t})]-\mathrm{P}[\mathrm{F}[\mathrm{t}+1] \mid>\text { Threshold }
$$

\section{B. Gaussian blur}

Next Gaussian blur is applied which is basically used to reduce the noise of the image. Blurring the image in the sense it also reduces the details of the image but the reduction of noise over weighs the reduction of detail and hence it is used. Gaussian smoothing is also used as a pre-processing stage in computer vision algorithms in order to enhance image structures at different scales. Mathematically, applying a Gaussian blur to an image is the same as convoluting the image with a Gaussian function. The Gaussian function is defined in equation (2).Here $\mathrm{x}, \mathrm{y}$ represent the pixel coordinates and $\mathrm{G}(\mathrm{x}, \mathrm{y})$ represents the function.

$$
G(x, y)=\frac{1}{2 \pi \sigma^{2}} e^{-\frac{x^{2}+y^{2}}{2 \sigma^{2}}}
$$

\section{Erosion}

Erosion (usually represented by $\Theta$ ) is one of two fundamental operations (the other being dilation) in image processing from which all other morphological operations are based. It was originally defined for binary images, later being extended to grayscale images, and subsequently to complete latices. Here we take the given binary image as input and a kernel/mask image of specific size. In our case it is a $5 * 5$ matrix of unity matrix and then mask the entire image to get the output. The below equation indicates that erosion of matrix A and B is the set of all points $\mathrm{z}$ such that B translated by $\mathrm{z}$ contained in A. Erosion is used to remove insignificant portion of the binary image like fluctuations, change in lighting condition.

$$
A \Theta B=\left\{z \in E \mid B_{Z} \subseteq A\right\}
$$

\section{Dilation}

Dilation is the process of magnifying a particular recognized object in the image this is done this algorithm is similar to erosion but here when comparing the kernel matrix with the binary matrix even if full match or partial match of kernel with the image happens it is considered as one. Dilation (usually represented by $\oplus$ ) is one of the basic operations in mathematical morphology. Originally developed for binary images, it has been expanded first to grayscale images, and then to complete latices. The dilation operation usually uses a structuring element for probing and expanding the shapes contained in the input image. The equation for dilation operation is given below. Dilation is used to magnify the significant part of the image i.e. moving object.

$$
A \oplus B=\bigcup_{b \in B} A_{b}
$$

\section{E. Contours}

After the binary image is processed and contains only the essential information it is now time to find contours. This is nothing but an edge detection algorithm which draws the boundaries around a moving object this is helpful in visualizing the part of the frame in which moving object is detected for this we are using chain approximation which is quite optimistic computationally for this purpose.

After finding the contours it is drawn in every frame of the image and displayed. If no motion is detected an empty array is returned based on this motion detected or no motion detected message is printed. Now that we know about contours, we have to make sure whether the contour is of reasonable size or not for this we find the largest contour in the frame. This is done by comparing the area of each contour in a particular frame. Now the centroid of the contour is calculated by using mid-point formula, a widely used mathematical formula for finding the mid-point of object in 2D plane.

\section{F. Multithreading}

We are using the $\mathrm{G}$ mail service for connecting to the server attaching the snapshot. The overhead when sending an email can be unpredictable as it depends on many factors like network connection, latency, server availability. So, this may slow down the performance. The system performance fell well below acceptable level due to the overhead of connecting to server establishing connection and sending a mail.

This problem is dealt using multithreading library and concurrently sending the email and processing the incoming stream of images A web-based alerting system was developed using SMTP API to send the mail to alert the user regarding suspicious activities. The average overhead of 1-2sec per 5 frames without multithreading was reduced to 
zero latency by using multithreading.

\section{Figures and Tabels}

In this section we will be presenting the block diagram for all the operation proposed in the algorithm above. Fig 1 presents the background subtraction algorithm. Fig 2 presents all the prepossessing morphological operations. Fig 3 and Fig 4 presents the email delivery system without and with multithreading.

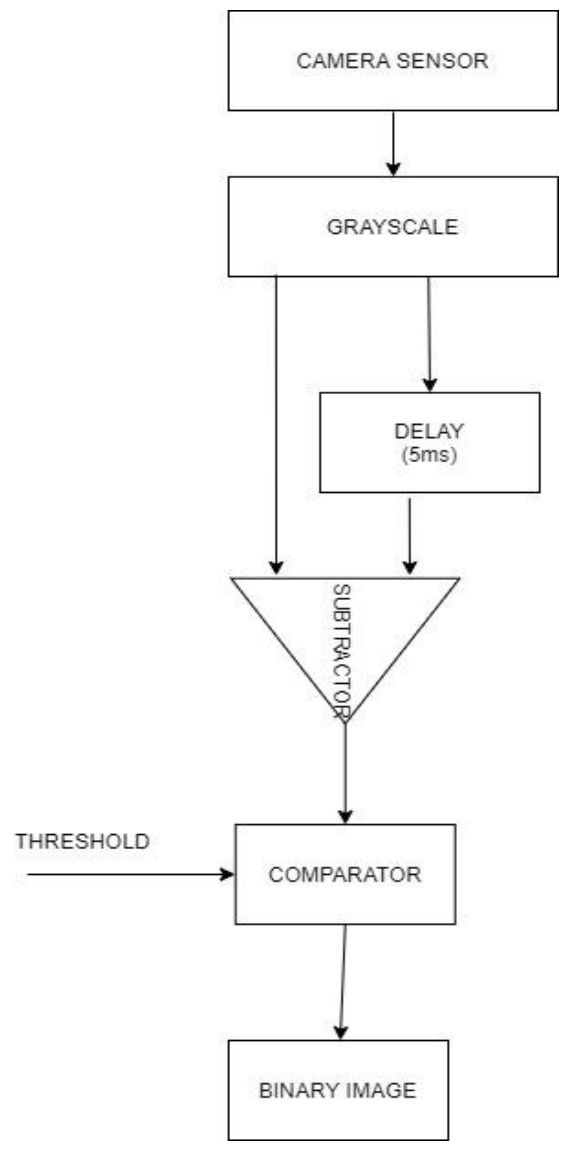

Fig.1. Background subtraction

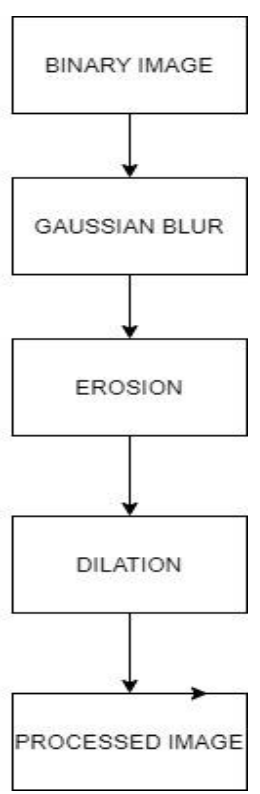

Fig.2. All pre-processing operations 


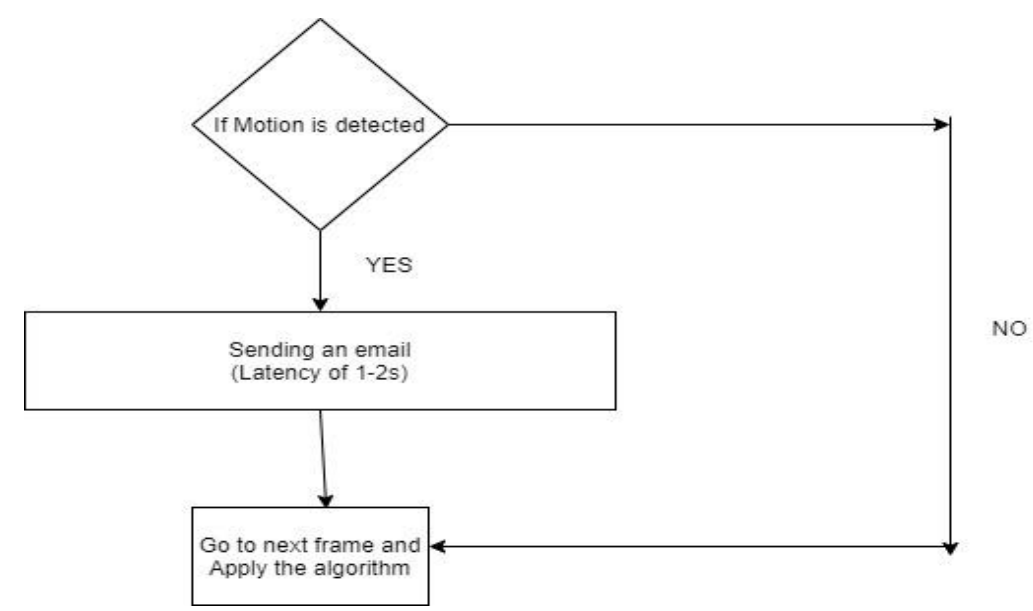

Fig.3. Without Multithreading

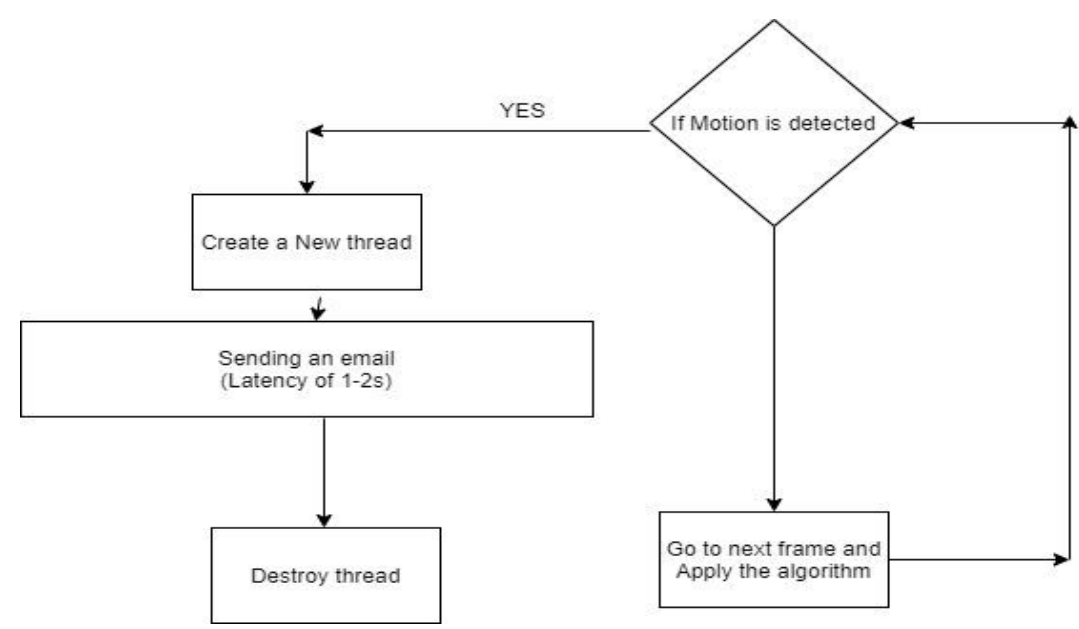

Fig.4. With using multithreading

\section{Results and Discussions}

\section{A. Motion Detection}

We got good results in terms of motion detection; Sensitivity of motion was optimal so it is detected only if significant motion has occurred. The threshold value for pixel variation was kept at 90 to 200 for a max value of 255 . And the area sensitivity was about 1400 pixels square for an image of dimension $720 * 1280$ in resolution.

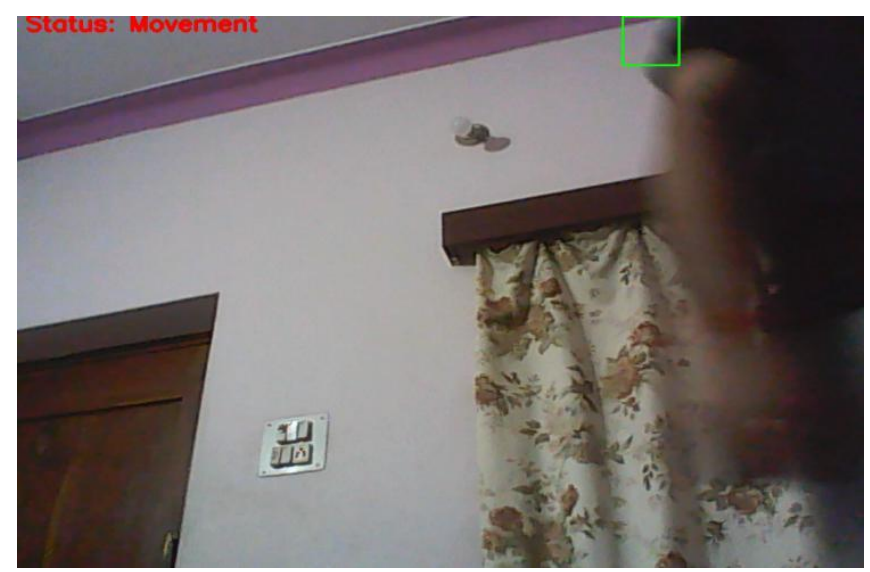




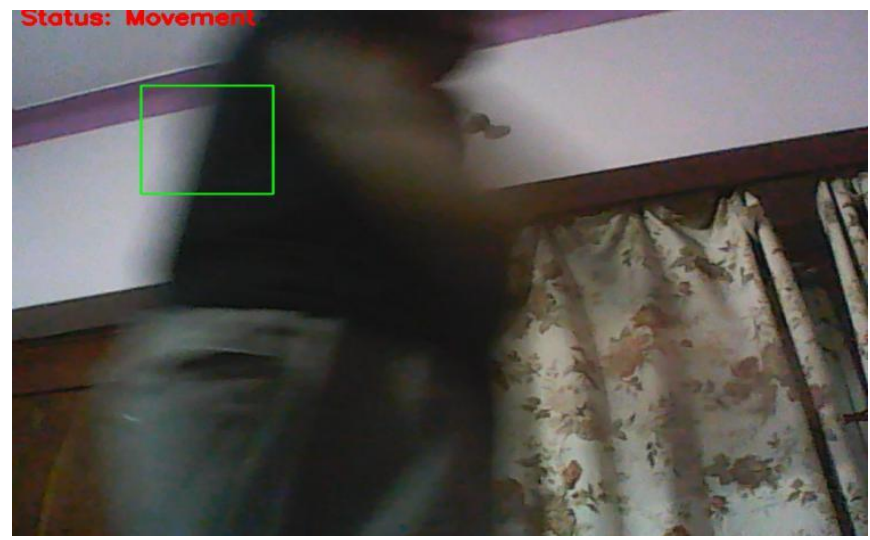

Fig.5. Movement

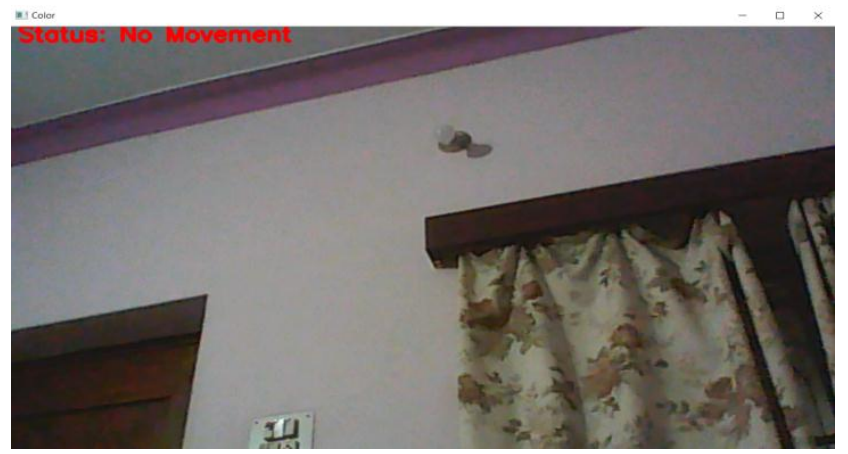

Fig.6. No Movement

\section{B. Direction detection}

Direction detection was implemented by comparing the centroid of the moving object in the subsequent frames if the centroid is greater in the next frame it is moving left else moving right, Due to lots of errors and ambiguity in this method. We mapped the horizontal pixel value of centroid of the pixel value to the angle of rotation of the servo the result of the above is tabulated below.

Table 1

\begin{tabular}{|c|c|c|}
\hline $\begin{array}{c}\text { Position of moving object } \\
\text { In horizontal }\end{array}$ & $\begin{array}{c}\text { ASCII value of 8-bit serial } \\
\text { code }\end{array}$ & Angle of servo rotation \\
\hline $\mathrm{x}<=100$ & 0 & 170 \\
\hline $\mathrm{x}>100$ and $\mathrm{x}<=200$ & 1 & 153 \\
\hline $\mathrm{x}>200$ and $\mathrm{x}<=300$ & 2 & 136 \\
\hline $\mathrm{x}>300$ and $\mathrm{x}<=400$ & 3 & 119 \\
\hline $\mathrm{x}>400$ and $\mathrm{x}<=500$ & 4 & 102 \\
\hline $\mathrm{x}>500$ and $\mathrm{x}<=600$ & 5 & 85 \\
\hline $\mathrm{x}>600$ and $\mathrm{x}<=700$ & 6 & 68 \\
\hline $\mathrm{x}>700$ and $\mathrm{x}<=800$ & 7 & 51 \\
\hline $\mathrm{x}>900$ and $\mathrm{x}<=1000$ & 8 & 34 \\
\hline $\mathrm{x}>1000$ and $\mathrm{x}<=1100$ & 9 & 17 \\
\hline
\end{tabular}

\section{Storage efficiency}

Here we got an average efficiency of 20 to $30 \%$. This efficiency will not only serve as a storage solution but also as an easy way of searching only the essential part of the video feed, so easy to find the most required part in the video. Due to the alarming rate in which data is getting generated in today's contemporary world. This serves as a vital aspect of storage efficiency 
Table 2

\begin{tabular}{l|l|l}
\hline \multicolumn{1}{c|}{ Trial no } & \multicolumn{1}{|c|}{$\begin{array}{c}\text { Original video size } \\
\text { (in MB) }\end{array}$} & \multicolumn{1}{|c}{$\begin{array}{c}\text { Optimized video size } \\
\text { (in MB) }\end{array}$} \\
\hline 1 & 10.52 & 8.38 \\
\hline 2 & 20 & 15.58 \\
\hline 3 & 5.2 & 3.52 \\
\hline 4 & 15.4 & 12.2 \\
\hline \multicolumn{2}{|c|}{ Trial no } & Efficiency (in \%) \\
\hline \multicolumn{2}{c|}{1} & 21.35 \\
\hline \multicolumn{2}{c|}{23.1} \\
\hline \multicolumn{2}{c|}{} & 33.3 \\
\hline \multicolumn{2}{c|}{4} & 21.8 \\
\hline
\end{tabular}

\section{Conclusion}

We have successfully designed and developed a video surveillance system that can track the direction of motion of an object and if two or more objects move simultaneously the object which has the larger dimension will be considered. And if the object is about to move out of the frame the camera moves accordingly so that the object in motion will be in the center of the frame. Also, the camera only records when it detects Motion so that storage redundancy can be eliminated and can be made highly efficient.The email delivery alerting system is also improved by using multithreading. This will advance the existing intrusion detection system to not only detect but track the moving object and also alert the user in real time.

\section{Acknowledgement}

We wish to express our heartily gratitude to Dr. Rohini Nagapadma, Ph.D., Principal, The National Institute of Engineering, Mysuru, India, for encouraging us to complete this accomplishment. We would like to thank our parents and also the faculty of, The National Institute of Engineering, Mysuru, India, for their valuable ideas, blueprint and support throughout the completion.

\section{References}

[1] Arghava, Keivani Jules-Raymon-Tapamo, Farzad Ghayoor "Motion-based Moving Object Detection and Tracking using Automatic K-means", IEEE African 2017 Proceedings.

[2] Shesha shah and P.S Sastry Published "Object tracking using motion direction detection" in Indian Institute of Science Bangalore.

[3] M Sahasri and C. Gireesh Published "Object motion Detection and tracking for video Surveillance" International journal of engineering trends and technology (IJETT) April 2017.

[4] Srinivasan.K, Porkumaran.K, and G. Sainarayan.2010." Improved Background subtraction Techniques for security in Video Applications".

[5] Kaman Kohli, Jatinder pal Singh and Anshul Kumar "Motion detection algorithm".

[6] Omar Elharrouss, Noor Al-Maadeed, Somaya Al-Maadeed "Motion Detection, Tracking and Classification for Automated Video Surveillance" Department of Computer Science and Engineering, Qatar University, IEEE Conference 2019.

[7] MANZANERA, Antoine et RICHEFEU, Julien C. A new motion detection algorithm based on $\Sigma-\Delta$ background estimation. Pattern Recognition Letters, 2007, vol. 28, no 3, p. 320-328.

[8] CHENG, Fan-Chieh, HUANG, Shih-Chia, et RUAN, Shanq-Jang. Illumination-sensitive background modeling approach for accurate moving object detection.Broadcasting, IEEE Transactions on, 2011, vol. 57, no 4, p. 794-801.

[9] WANG, Yong, LU, Qian, WANG, Dianhong, et al. Compressive background modeling for foreground extraction. Journal of Electrical and Computer Engineering, 2015, vol. 2015, p. 13.

[10] LIN, Dazhen, CAO, Donglin, et ZENG, Hualin. Improving motion state change object detection by using block background context. In : Computational Intelligence (UKCI), 2014 14th UK Workshop on. IEEE, 2014. p. 1-6.

[11] GHAEMINIA, Mohammad Hossein et SHOKOUHI, Shahryar Baradaran. Adaptive background model for moving objects based on PCA. In : Machine Vision and Image Processing (MVIP), 2010 6th Iranian. IEEE, 2010. p. 1-4.

[12] LIAO, Shengcai, ZHAO, Guoying, KELLOKUMPU, Vili, et al. Modeling pixel process with scale invariant local patterns for background subtraction in complex scenes. In : Computer Vision and Pattern Recognition (CVPR), 2010 IEEE Conference on. IEEE, 2010. p. 1301-1306.

[13] M. Botha, R. Solms, "Utilizing Neural Networks For Effective Intrusion Detection", ISSA, 2004.

[14] Anup Goyal, Chetan Kumar, "GA-NIDS: A Genetic Algorithm based Network Intrusion Detection System”, 2008.

[15] Lee W., Stolfo S., and Mok K., “Adaptive Intrusion Detection: A Data Mining Approach,” Artificial Intelligence Review, 14(6), December 2000, pp. 533-567. 
[16] Stolfo J., Fan W., Lee W., Prodromidis A., and Chan P.K., "Cost-based modeling and evaluation for data mining with application to fraud and intrusion detection,” DARPA Information Survivability Conference, 2000.

\section{Authors' Profiles}

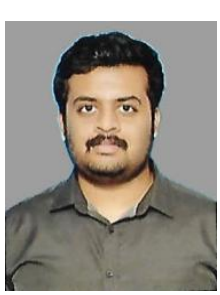

Adithya Urs completed his B.E in Electronics and Communication from The National Institute of Engineering Mysuru. He has also done 6 months of internship in Robert Bosch engineering and business solution pvt

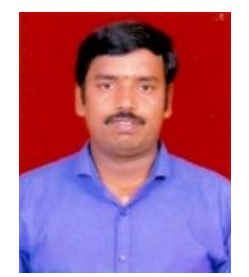

Dr. C Nagaraju completed his B.E and M-Tech from SJCE, Mysore. He has completed his Ph.D. at PET Research centre, Mandya. He has 15 years of teaching experience and currently working as Assistant Professor, in department of ECE at The National Institute of Engineering, Mysore. He has published more than 30 research publications in various National, International journals and Conference proceedings.

How to cite this paper: Adithya Urs, Nagaraju C, " Object Motion Direction Detection and Tracking for Automatic Video Surveillance", International Journal of Education and Management Engineering (IJEME), Vol.11, No.2, pp. 32-39, 2021. DOI: 10.5815/ijeme.2021.02.04 